\title{
Flächeninanspruchnahme 2020 und das 30-ha-Ziel: Regionale Verteilungsschlüssel und Anpassungserfordernisse
}

\author{
Ralph Henger $\cdot$ Christoph Schröter-Schlaack $\cdot$ \\ Philip Ulrich • Martin Distelkamp
}

Eingegangen: 8. Dezember 2009 / Angenommen: 14. Mai 2010 / Online publiziert: 17. Juli 2010

(C) Springer-Verlag 2010

\begin{abstract}
Zusammenfassung Die Reduzierung der Flächeninanspruchnahme sowie die Umsetzung des 30-ha-Ziels der nationalen Nachhaltigkeitsstrategie erfordert Anpassungen aller Bundesländer, Regionen und Kommunen. Eine Regionalisierung dieser siedlungspolitischen Zielvorgabe steht bis heute allerdings noch aus. Dieser Beitrag präsentiert daher ein bundeseinheitliches Zuteilungsverfahren, welches quantitative Flächensparziele für Gebietskörperschaften mit den Anteilen an der Bevölkerung und der Katasterfläche bestimmt. Die sich aus diesem Bevölkerungs-Fläche-Indikator (BeFla-Indikator) ergebenden Verteilungsmuster werden auf Ebene der Bundesländer und Raumordnungsregionen diskutiert. Hierfür erfolgt eine Gegenüberstellung der abgeleiteten Mengenvorgaben mit der vergangenen und zukünftigen Flächeninanspruchnahme. Für die zukünftige Siedlungsentwicklung werden die Simulationen des Modells Panta Rhei Regio herangezogen, welche die Entwicklung der Flächeninanspruchnahme für
\end{abstract}

\footnotetext{
R. Henger $(\square)$

Wirtschaftspolitik und Mittelstandsforschung, Georg-August

Universität Göttingen, Platz der Göttinger Sieben 3,

37073 Göttingen, Deutschland

E-Mail: ralph.henger@wiwi.uni-goettingen.de

C. Schröter-Schlaack

Department Ökonomie, Helmholtz-Zentrum für

Umweltforschung - UFZ, Permoserstraße 15,

04318 Leipzig, Deutschland

E-Mail: christoph.schroeter-schlaack@ufz.de

P. Ulrich · M. Distelkamp

Gesellschaft für Wirtschaftliche Strukturforschung,

Heinrichstraße 30, 49080 Osnabrück, Deutschland

E-Mail: ulrich@gws-os.de

M. Distelkamp

E-Mail: distelkamp@gws-os.de
}

Siedlung und Verkehr bis 2020 auf Kreisebene projizieren. Die Prognosen lassen auf ein deutlich moderateres, aber weiterhin stetiges Siedlungsflächenwachstum schließen, welches die weiterhin hohe Notwendigkeit quantitativer Zielvorgaben und ihre Relevanz zur Umsetzung einer nachhaltigen Siedlungsentwicklung unterstreicht. Vor diesem Hintergrund ermöglichen die Aufteilung des 30-haZiels und die Gegenüberstellung von Bedarf und zulässiger Menge eine fundierte Diskussion über die konkreten Anpassungserfordernisse und notwendigen Anstrengungen der einzelnen Länder und Regionen.

Schlüsselwörter Siedlungsentwicklung · Modell · Projektion · 30-ha-Ziel $\cdot$ Regionale Nachhaltigkeit

\section{Land Consumption 2020 and the National 30-ha-target: Regional Distribution and Adjustments}

Abstract The reduction of land development and the attainment of the national 30-ha-sustainability-target require all states, regions and municipalities in Germany to adjust. Up to now, however, regional targets for local jurisdictions have not been achieved. This paper presents a top-down mechanism by which a national target is subdivided according to the population and total area shares of each administrative unit. Distributional implications of this rule are analyzed on the level of states and regions. For this purpose, the derived sub-quantities are compared with the past and future land development rates. The future development of land consumption is estimated by the Panta Rhei Regio model, which projects growth rates of settlement and traffic areas until 2020. The results show that although land development will decline significantly in the next years, it will still clearly remain above the 30-ha-target level. As 
the simulation is modeled on county level the results also indicate the need to specify detailed quantity standards at regional level. A definition of subdivided targets for states, regions and municipalities offers the opportunity to improve the ongoing German debate on open space preservation and to initiate greater efforts on the way to a more efficient use of natural resource land.

Keywords Land development $\cdot$ Model Projection 30-ha-target $\cdot$ Regional sustainability

\section{Einleitung}

Die Forderung nach einer Begrenzung der Flächeninanspruchnahme für Siedlungs- und Verkehrszwecke und der Integration quantitativer Zielstellungen in das Planungsrecht waren in den letzten Jahren beherrschende Themen in der Diskussion um die räumliche Entwicklung in Deutschland. Die negativen ökologischen, ökonomischen und auch sozialen Konsequenzen einer beständig auf Zuwachs und nach außen ausgerichteten Siedlungsentwicklung führten zu der Aufnahme des 30-ha-Ziels in die Nationale Nachhaltigkeitsstrategie der damaligen Bundesregierung im Jahr 2002 (vgl. Bundesregierung 2002; Wissenschaftlicher Beirat Bodenschutz 2002; Umweltbundesamt 2004). ${ }^{1}$ Demnach soll die Flächeninanspruchnahme für Siedlungs- und Verkehrszwecke von aktuell gut 100 auf 30 ha je Tag bis zum Jahr 2020 reduziert werden. Dieses ,30-ha-Ziel“ hat einerseits intensive Diskussionen um das Für und Wider solcher konkreten „Flächenverbrauchsziele“ ausgelöst, andererseits die Debatte um die Reform bestehender bzw. die Einführung neuartiger Instrumente zur Beeinflussung der Flächeninanspruchnahme weiter angefacht (vgl. Apel/Henckel/Bunzel et al. 1995; Bizer/Bunzel/Cichorowski et al. 2006; Köck/ Bizer/Einig et al. 2008; Ring 2008; Davy 2009).

Ungeachtet seines ambitionierten Charakters, die Flächeninanspruchnahme in nunmehr noch zehn Jahren bis 2020 um mehr als zwei Drittel zu verringern, sind die konkreten Folgen einer Umsetzung des 30-ha-Ziels für die betroffenen Länder, Regionen und Kommunen jedoch noch weitgehend unerforscht. ${ }^{2}$ Dadurch wird eine zielführende Diskussion um Sinn und Umsetzbarkeit des 30-ha-Ziels

\footnotetext{
${ }^{1}$ Die Große Koalition übernahm das 30-ha-Ziel im Jahr 2005. Der Koalitionsvertrag der aktuellen Regierung hat von einer konkreten Zielvorgabe allerdings Abstand genommen, betont aber weiterhin den Grundsatz „Innen- vor Außenentwicklung“ und beabsichtigt die Erprobung „überregionaler Handelssysteme für die Flächennutzung“ (Bundesregierung 2009: $31 \mathrm{f}$.).

${ }^{2}$ Eine Ausnahme bildet hierbei das Papier von Jakubowski und Zarth (2003), die Anpassungslasten deutscher Regionen an eine deutlich verknappte Flächenausweisung anhand der Dominanz bestimmter Industrie- und Gewerbezweige zumindest qualitativ abschätzen.
}

erheblich belastet. Das Fehlen konkreter Zielaufteilungsmöglichkeiten und der Untersuchung daraus resultierender Anpassungserfordernisse hat mehrere Ursachen: Quantitative (und somit konkrete) Zielvorgaben werden im kommunalpolitischen Raum überwiegend abgelehnt, da diese als ein zu starker Eingriff in die kommunale Planungshoheit verstanden werden und negative Effekte auf die wirtschaftliche Entwicklung und die Wohnversorgung befürchtet werden (Köck/Bizer/Einig et al. 2008: 30 und 56 ff.). Daneben besteht heftiger Widerstreit über die zukünftig zu erwartende Entwicklung der Flächeninanspruchnahme für Siedlungsund Verkehrszwecke und somit über die Konsequenzen quantitativer Reduktionsziele. Verschiedene Interessengruppen formulieren äußerst unterschiedliche und von den jeweiligen institutionellen Hintergründen beeinflusste Prognosen. Einen Pol bildet der optimistische Glaube an eine „heilende“ Selbstregulierung aus Bevölkerungsrückgang und verbesserter Transparenz vor allem der (ökonomischen) Folgen bauleitplanerischer Flächennutzungsentscheidungen. Flächenreduktionsziele, die sich am 30-ha-Ziel orientieren, wären somit obsolet. Die Gegenposition befürchtet einen sich noch verstärkenden kommunalen Wettbewerb um Einwohner und Gewerbeansiedlungen mit kostengünstigem Bauland und sieht in den ersten zögerlichen Verbesserungen in der Statistik noch keinesfalls die erhoffte „Trendwende“ bei der Flächeninanspruchnahme. Darüber hinaus mangelt es an konkreten Vorschlägen, wie das bundesweite 30-haZiel auf die eigentlichen Entscheidungsträger im Rahmen der kommunalen und regionalen Bauleitplanung umgesetzt werden kann. Dies mag auch an der nicht vorhandenen Gesetzgebungskompetenz des Bundes hinsichtlich eines solchen (flächen-)entwicklungspolitischen Ziels liegen, da die Planungshoheit in der Bundesrepublik den Bundesländern zufällt (für einen Überblick über die aktuelle Kompetenzlage vgl. Bovet/Köck 2008). Allerdings führt das Fehlen konkreter Allokationsvorschläge auch zu Interpretationsmöglichkeiten, die die möglichen Anpassungslasten für Regionen und Kommunen in Deutschland verschleiern. In der Folge wird das 30-ha-Ziel entweder generell als zu strikt und nicht umsetzbar abgelehnt oder aber es werden die Anpassungsnotwendigkeiten vornehmlich „bei den Anderen" gesehen (Bizer/Cichorowski/Henger et al. 2009).

Vor diesem Hintergrund versucht der vorliegende Beitrag die Diskussion um das 30-ha-Ziel und seine möglichen Folgen mit zwei neuartigen Ansätzen zu bereichern und zu versachlichen. Ausgehend von einer Analyse der bisherigen Siedlungsentwicklung (Kap. 2) wird einerseits die zukünftige Siedlungsentwicklung mittels des Modells Panta Rhei Regio (PRR) abgeschätzt (Kap. 3). Dieses Modell bietet einen systematischen und analytischen Weg zur Bestimmung der zukünftigen Siedlungsentwicklung auf Kreisebene. Zusätzlich wird eine Methode zur Aufteilung der innerhalb des Sparziels noch zulässigen Flächeninan- 
Tab. 1 Entwicklung der Siedlungs- und Verkehrsfläche (SuV) 1996 bis 2008

\begin{tabular}{|c|c|c|c|c|c|c|}
\hline \multirow[t]{3}{*}{ Gebiet } & \multicolumn{6}{|c|}{ Veränderung 1996-2008 } \\
\hline & \multicolumn{3}{|c|}{ Siedlungs- und Verkehrsfläche } & \multicolumn{3}{|c|}{ Anteil am Zuwachs SuV } \\
\hline & $\mathrm{km}^{2}(1996)$ & $\begin{array}{l}\text { Relative } \\
\text { Änderung (\%) }\end{array}$ & $\begin{array}{l}\text { ha pro } \\
\text { Tag }\end{array}$ & $\begin{array}{l}\text { Gebäude- und } \\
\text { Freifläche (\%) }\end{array}$ & $\begin{array}{l}\text { Übrige Siedlungs- } \\
\text { flächen }(\%)\end{array}$ & $\begin{array}{l}\text { Verkehrs- } \\
\text { fläche }(\%)\end{array}$ \\
\hline Alte Bundesländer & $32.649,4$ & 10,5 & 78,0 & 60,8 & 18,4 & 20,8 \\
\hline Berlin, Brandenburg \& Thüringen & $4.239,4$ & 12,6 & 12,2 & 47,6 & 32,5 & 19,9 \\
\hline $\begin{array}{l}\text { Mecklenburg-Vorpommern, Sachsen \& } \\
\text { Sachsen-Anhalt }\end{array}$ & $5.163,4$ & 21,7 & 25,5 & 12,3 & 71,2 & 16,5 \\
\hline $\begin{array}{l}\text { Sachsen-Anhalt } \\
\text { Deutschland }\end{array}$ & $42.052,2$ & 12,1 & 115,8 & 48,7 & 31,6 & 19,8 \\
\hline
\end{tabular}

Quelle: Statistisches Bundesamt (2009)

spruchnahme auf die Raumordnungsregionen der Bundesrepublik Deutschland vorgestellt (Kap. 4). Die Berechnung des „BeFla-Indikators“ durch eine Gleichgewichtung der Kriterien Bevölkerung und Katasterfläche stellt einen bundeseinheitlich anwendbaren Vergleichsmaßstab und Verteilungsschlüssel zur Verfügung. Aus der Zusammenschau von prognostizierter zukünftiger Flächeninanspruchnahme und der mittels BeFla-Indikator bestimmten Obergrenze zulässiger Siedlungsentwicklung ergeben sich konkrete Anpassungserfordernisse von Bundesländern und Regionen zur Umsetzung des 30-ha-Ziels. Hierdurch wird auch eine neue Argumentationsgrundlage in der Diskussion um die instrumentelle Umsetzung des 30-ha-Ziels geschaffen. Auf Basis der vorgenommenen Berechnungen können Vor- und Nachteile einzelner instrumenteller Umsetzungswege detailliert beschrieben werden. Dies ermöglicht einen Beitrag zur Versachlichung dieser Diskussion.

\section{Bisherige Siedlungsentwicklung}

Flächennutzung und Siedlungsentwicklung werden in Deutschland statistisch durch die amtliche Flächenerhebung nach Art der tatsächlichen Nutzung erfasst (Deggau 2006). Diese Datenquelle wird alle vier Jahre bundesweit erhoben und seit 2001 auch jährlich für Siedlungs- und Verkehrsflächen fortgeschrieben. Dieses Kapitel beschreibt die Entwicklung der Siedlungs- und Verkehrsflächen sowie der Gebäude- und Freiflächen zwischen den Jahren 1996, 2000, 2004 und 2008. Dabei sollen nicht nur grundlegende Trends in einer aggregierten Betrachtung identifiziert, sondern auch prägnante Muster der Flächeninanspruchnahme im regionalen Kontext aufgedeckt werden. Dabei erfolgt zwangsläufig die Auseinandersetzung mit statistischen Ungenauigkeiten, die mit abnehmender Aggregation erheblich zunehmen.

In Tab. 1 ist zunächst die Entwicklung der Siedlungsund Verkehrsflächen über den gesamten Zeitraum von 1996 bis 2008 dargestellt. Dabei wurden die Siedlungs- und Verkehrsflächen zur Veranschaulichung in drei Kategorien aufgeteilt: Gebäude- und Freiflächen, sonstige Siedlungsflächen (Betriebsflächen, Erholungsflächen und Friedhofs- flächen) und Verkehrsflächen. Die Neuinanspruchnahme von Flächen für Siedlung und Verkehr belief sich im betrachteten Zeitraum auf durchschnittlich knapp 116 ha pro Tag. Mit Blick auf den relativen Zuwachs der Fläche ist die Dynamik in den neuen Bundesländern etwas stärker, auch wenn die durch erhebliche statistische Verwerfungen geprägten Bundesländer ausgeschlossen werden. ${ }^{3}$ Etwa die Hälfte der Flächeninanspruchnahme kam durch die Ausweitung der Gebäude- und Freiflächen zustande. Mit etwa $20 \%$ ist die Neuinanspruchnahme für Verkehrszwecke ein weiterer Treiber des Freiflächenverlustes gewesen. Unter den übrigen Siedlungsflächen sind die Erholungsflächen die bedeutendste Flächennutzung mit Blick auf den Flächenverbrauch. Die Flächeninanspruchnahme für Friedhofsfläche und Betriebsfläche (ohne Abbauland) fällt mit 6 bis $7 \%$ des Zuwachses nur gering ins Gewicht. Der Schwerpunkt bei den „Nutzern“, soweit die Auswertung aus der Statistik möglich war, lag im Zeitraum von 1996 bis 2004 im Bereich der Gebäude- und Freiflächen auf dem Bereich „Wohnen“. Die Ursachen liegen u. a. im Nachfragerückgang für die Gewerbeflächen im Zuge der schwachen konjunkturellen Entwicklung zwischen 2000 und 2004.

Unabhängig von statistischen Ungenauigkeiten kann man mit Blick auf die Vier-Jahres-Flächenstatistik (vgl. historische Entwicklung laut Statistik in Tab. 2) eine langsame, jedoch kontinuierliche Reduktion der Flächeninanspruchnahme für Siedlung und Verkehr beobachten. Einem Rückgang der Flächeninanspruchnahme von $20 \%$ in den alten Bundesländern steht eine Reduktion von nur $10 \%$ zwischen dem Zeitraum 1996 bis 2000 und 2004 bis 2008 in den neuen Bundesländern gegenüber. Der Vergleich mit der Entwicklung der Gebäude- und Freifläche zeigt jedoch, dass in einigen Bundesländern die Umschlüsselungsproblematik (vgl. Fußnote 3) im Bereich der Flächenerhebung fortbesteht (vgl. Deggau 2006; Siedentop/Heiland/Lehmann et al. 2007).

\footnotetext{
${ }^{3}$ Mecklenburg-Vorpommern, Sachsen, Sachsen-Anhalt sind die Länder, in denen die Umstellung der Erhebungssystematik im betrachteten Zeitraum auf Ebene der Bundesländer eine besonders starke Wirkung zeigt (vgl. Deggau 2006).
} 
Tab. 2 Entwicklung der Siedlungs- und Verkehrsfläche nach Bundesländern

\begin{tabular}{|c|c|c|c|c|c|c|c|c|c|c|c|c|c|}
\hline \multirow[t]{3}{*}{ Bundesland } & \multirow[t]{3}{*}{ Bestand $1996\left(\mathrm{~km}^{2}\right)$} & \multicolumn{6}{|c|}{ Historische Entwicklung laut Statistik ${ }^{\mathrm{a}}$} & \multicolumn{6}{|c|}{ Projektion mit PANTA RHEI REGIO $^{b}$} \\
\hline & & \multicolumn{2}{|c|}{1996 bis 2000} & \multicolumn{2}{|c|}{2000 bis 2004} & \multicolumn{2}{|c|}{2004 bis 2008} & \multicolumn{2}{|c|}{2008 bis 2012} & \multicolumn{2}{|c|}{2012 bis 2016} & \multicolumn{2}{|c|}{2016 bis 2020} \\
\hline & & $\begin{array}{l}\text { ha pro } \\
\text { Tag }\end{array}$ & $\%$ & $\begin{array}{l}\text { ha pro } \\
\text { Tag }\end{array}$ & $\%$ & $\begin{array}{l}\text { ha pro } \\
\text { Tag }\end{array}$ & $\%$ & $\begin{array}{l}\text { ha pro } \\
\text { Tag }\end{array}$ & $\%$ & $\begin{array}{l}\text { ha pro } \\
\text { Tag }\end{array}$ & $\%$ & $\begin{array}{l}\text { ha pro } \\
\text { Tag }\end{array}$ & $\%$ \\
\hline Schleswig-Holstein & $1.699,9$ & 4,1 & 3,6 & 8,4 & 7,0 & 5,4 & 4,2 & 4,2 & 3,1 & 3,7 & 2,7 & 3,4 & 2,4 \\
\hline Hamburg & 427,2 & 0,2 & 0,8 & 0,8 & 2,7 & 0,5 & 1,6 & 0,6 & 1,8 & 0,5 & 1,7 & 0,5 & 1,6 \\
\hline Niedersachsen & $5.783,5$ & 16,4 & 4,1 & 14,4 & 3,5 & 11,6 & 2,7 & 10,4 & 2,4 & 8,9 & 2,0 & 8,3 & 1,8 \\
\hline Bremen & 218,0 & 0,5 & 3,6 & 0,2 & 1,1 & 0,2 & 1,4 & 0,1 & 0,9 & 0,1 & 0,6 & 0,1 & 0,4 \\
\hline Nordrhein-Westfalen & $6.906,9$ & 16,5 & 3,5 & 15,2 & 3,1 & 14,2 & 2,8 & 6,8 & 1,3 & 5,8 & 1,1 & 5,3 & 1,0 \\
\hline Hessen & $3.067,9$ & 4,9 & 2,3 & 3,9 & 1,8 & 3,6 & 1,6 & 3,5 & 1,6 & 3,3 & 1,5 & 3,2 & 1,4 \\
\hline Rheinland-Pfalz & $2.577,2$ & 5,4 & 3,1 & 5,8 & 3,2 & 5,4 & 2,9 & 2,7 & 1,4 & 2,5 & 1,3 & 2,4 & 1,2 \\
\hline Baden-Württemberg & $4.542,9$ & 12,0 & 3,9 & 10,4 & 3,2 & 9,2 & 2,8 & 9,5 & 2,8 & 8,4 & 2,4 & 7,8 & 2,2 \\
\hline Bayern & $6.929,7$ & 28,4 & 6,0 & 18,0 & 3,6 & 17,2 & 3,3 & 14,1 & 2,6 & 13,2 & 2,4 & 12,5 & 2,2 \\
\hline Saarland & 496,1 & 0,7 & 2,0 & 0,7 & 2,1 & 0,6 & 1,6 & 0,5 & 1,4 & 0,4 & 1,1 & 0,3 & 0,9 \\
\hline $\begin{array}{l}\text { Alte Bundesländer } \\
\text { (ohne Berlin) }\end{array}$ & 32.649 & 89,1 & 4,0 & 77,8 & 3,3 & 67,8 & 2,8 & 52,4 & 2,1 & 47,0 & 1,9 & 43,9 & 1.7 \\
\hline Berlin & 594,4 & 1,4 & 3,5 & 0,3 & 0,7 & 0,3 & 0,6 & 1,3 & 2,9 & 0,9 & 2,0 & 0,7 & 1,6 \\
\hline Brandenburg & $2.279,9$ & 9,3 & 6,0 & 8,2 & 4,9 & 9,5 & 5,5 & 4,6 & 2,5 & 4,1 & 2,2 & 4,0 & 2,1 \\
\hline Mecklenburg-Vorpommern & $1.440,6$ & 7,6 & 7,7 & 8,7 & 8,2 & 7,8 & 6,8 & 2,5 & 2,1 & 2,4 & 1,9 & 2,1 & 1,7 \\
\hline Sachsen & $1.952,5$ & 8,2 & 6,2 & 5,2 & 3,6 & 6,4 & 4,4 & 3,9 & 2,6 & 3,5 & 2,3 & 3,4 & 2,2 \\
\hline Sachsen-Anhalt & $1.770,3$ & 9,9 & 8,1 & 12,8 & 9,8 & 10,0 & 7,0 & 3,1 & 2,0 & 2,6 & 1,7 & 2,3 & 1,5 \\
\hline Thüringen & $1.365,1$ & 3,6 & 3,9 & 2,2 & 2,2 & 1,9 & 2,0 & 1,7 & 1,7 & 1,5 & 1,5 & 1,5 & 1,4 \\
\hline $\begin{array}{l}\text { Neue Bundesländer } \\
\text { (inkl. Berlin) }\end{array}$ & 9.403 & 40,0 & 6,2 & 37,3 & 5,5 & 36,0 & 5,0 & 17,0 & 2,3 & 14,0 & 2,0 & 14,0 & 1,8 \\
\hline Deutschland & 42.052 & 129,1 & 4,5 & 115,1 & 3,8 & 103,8 & 3,3 & 69,4 & 2,2 & 61,0 & 1,9 & 57,9 & 1,7 \\
\hline
\end{tabular}

${ }^{\mathrm{a}}$ Quelle: Statistisches Bundesamt (2009)

${ }^{b}$ Quelle: PANTA RHEI REGIO Referenzszenario (9/2009)

In Tab. 3 ist zu erkennen, dass bei den Gebäude- und Freiflächen besonders hohe relative Zuwächse auf Länderebene in Schleswig-Holstein, Niedersachsen, Bayern, Brandenburg und Sachsen-Anhalt zu beobachten waren. Etwa $55 \%$ der Flächeninanspruchnahme für Gebäude- und Freifläche der jüngeren Vergangenheit entfallen auf diese Bundesländer. Dagegen waren beispielsweise in den Stadtstaaten nur wenige Veränderungen zu beobachten. Die Ergebnisse für den Zeitraum 2004 bis 2008 lassen erkennen, dass Bayerns Wachstum weiter anhält, während sich die Entwicklungen z. B. in Schleswig-Holstein und Brandenburg abschwächen. Der Rückgang des Flächenzuwachses bei Gebäudeund Freiflächen setzt sich nach derzeitigen Erkenntnissen seit 2004 fort, jedoch wird er, sofern die Flächenrückgänge in Sachsen-Anhalt und Mecklenburg-Vorpommern außer Betracht gelassen werden, etwas gebremst.

Eine Gegenüberstellung der Siedlungs- und Verkehrsfläche mit der Gebäude- und Freifläche zeigt, dass deren Entwicklungen weitgehend parallel verlaufen. Nur in Schleswig-Holstein, Mecklenburg-Vorpommern und Sachsen-Anhalt haben sich die Anteile der Gebäude- und Freiflächen verschoben. Ansonsten bleibt die Verschiebung des Anteils im Zeitraum 1996 bis 2004 zwischen -1,3 und +1,4 Prozentpunkten. Im Folgenden wird die Analyse der Flächeninanspruchnahme auf die Gebäude- und Freifläche eingeschränkt. Dies hat folgende Gründe: 1) Zum einen bestätigt sich auch auf Kreisebene, dass die Expansion der eigentlichen „Siedlungsbereiche“ (Gebäude- und Freifläche) für die gesamte Siedlungsentwicklung maßgeblich ist und daher auch in vergleichbaren Analysen nur auf die Gebäude- und Freifläche zurückgegriffen wird (Umweltbundesamt 2003; Bundesamt für Bauwesen und Raumordnung 2007; Dosch 2008). Dies hat außerdem 2) mit dem höheren Grad an statistischen Ungenauigkeiten bei den übrigen Siedlungsflächen (insbesondere Erholungsflächen) zu tun. Des Weiteren 3) ist die Aussagekraft der Ergebnisse des Modells Panta Rhei Regio im regionalen Kontext im Bereich der Erholungsund Verkehrsflächen eingeschränkt. Ein weiteres Argument für die Einschränkung der Analyse auf die Gebäude- und Freiflächen ist 4) die Einschätzung, dass Anpassungserfordernisse meist vor allem auf kommunaler Ebene diskutiert werden sollten. Einige Flächennutzungsänderungen, wie etwa bei Errichtung neuer überörtlicher Straßen, haben ihren Ursprung jedoch nicht in den Entscheidungen kommunaler Akteure. Dies bedeutet nicht, dass diese Flächen für die Beurteilung der Anpassungserfordernisse vernachlässigbar sind. Vieles deutet sogar darauf hin, dass die Bedeutung von Verkehrsflächen bei der Siedlungs- und Verkehrsflächenentwicklung bis 2020 zunehmen wird (Distelkamp/Großmann/ Hohmann et al. 2009). Gebäude- und Freiflächen erlauben jedoch deutlich präzisere Angaben zu Ursache und Wirkung flächenrelevanter Entwicklungen. 
Tab. 3 Entwicklung der Gebäude- und Freifläche nach Bundesländern

\begin{tabular}{|c|c|c|c|c|c|c|c|c|c|c|c|c|c|}
\hline \multirow[t]{3}{*}{ Bundesland } & \multirow[t]{3}{*}{ Bestand $1996\left(\mathrm{~km}^{2}\right)$} & \multicolumn{6}{|c|}{ Histori sche Entwicklung laut Statistik ${ }^{\mathrm{a}}$} & \multicolumn{6}{|c|}{ Projektion mit PANTA RHEI REGIO $^{b}$} \\
\hline & & \multicolumn{2}{|c|}{1996 bis 2000} & \multicolumn{2}{|c|}{2000 bis 2004} & \multicolumn{2}{|c|}{2004 bis 2008} & \multicolumn{2}{|c|}{$2008-2012$} & \multicolumn{2}{|c|}{$2012-2016$} & \multicolumn{2}{|c|}{$2016-2020$} \\
\hline & & $\begin{array}{l}\text { ha pro } \\
\text { Tag }\end{array}$ & $\%$ & $\begin{array}{l}\text { ha pro } \\
\text { Tag }\end{array}$ & $\%$ & $\begin{array}{l}\text { ha pro } \\
\text { Tag }\end{array}$ & $\%$ & $\begin{array}{l}\text { ha pro } \\
\text { Tag }\end{array}$ & $\%$ & $\begin{array}{l}\text { ha pro } \\
\text { Tag }\end{array}$ & $\%$ & $\begin{array}{l}\text { ha pro } \\
\text { Tag }\end{array}$ & $\%$ \\
\hline Schleswig-Holstein & 900,7 & 4,9 & 7,9 & 6,1 & 9,2 & 1,6 & 2,3 & 2,6 & 3,4 & 2,3 & 2,9 & 2,0 & 2,5 \\
\hline Hamburg & 266,8 & 0,1 & 0,7 & 0,4 & 2,3 & 0,4 & 2,0 & 0,4 & 2,2 & 0,4 & 2,0 & 0,4 & 2,0 \\
\hline Niedersachsen & $3.027,4$ & 11,2 & 5,4 & 9,4 & 4,3 & 7,4 & 3,3 & 6,2 & 2,6 & 5,0 & 2,1 & 4,6 & 1,9 \\
\hline Bremen & 133,8 & 0,2 & 1,7 & 0,2 & 1,9 & 0,0 & 0,5 & 0,1 & 1,0 & 0,1 & 0,6 & 0,0 & 0,4 \\
\hline Nordrhein-Westfalen & $4.033,1$ & 9,5 & 3,4 & 7,1 & 2,5 & 4,4 & 1,5 & 3,9 & 1,3 & 3,3 & 1,1 & 2,9 & 1,0 \\
\hline Hessen & $1.470,7$ & 3,3 & 3,2 & 2,1 & 2,0 & 2,1 & 2,0 & 2,0 & 1,8 & 1,8 & 1,7 & 1,7 & 1,6 \\
\hline Rheinland-Pfalz & $1.030,7$ & 3,6 & 5,1 & 3,5 & 4,7 & 2,6 & 3,4 & 1,3 & 1,6 & 1,2 & 1,5 & 1,1 & 1,3 \\
\hline Baden-Württemberg & $2.374,2$ & 8,6 & 5,3 & 6,8 & 4,0 & 5,6 & 3,2 & 6,0 & 3,3 & 5,2 & 2,8 & 4,8 & 2,5 \\
\hline Bayern & $3.398,3$ & 18,1 & 7,8 & 11,0 & 4,4 & 11,2 & 4,3 & 8,3 & 3,1 & 7,7 & 2,8 & 7,2 & 2,5 \\
\hline Saarland & 294,7 & 0,5 & 2,3 & 0,5 & 2,5 & 0,4 & 1,9 & 0,3 & 1,3 & 0,2 & 1,1 & 0,2 & 0,8 \\
\hline $\begin{array}{l}\text { Alte Bundesländer } \\
\text { (ohne Berlin) }\end{array}$ & 16.931 & 59,8 & 5,2 & 47,1 & 3,9 & 35,9 & 2,8 & 31,0 & 2,4 & 27,2 & 2,0 & 25,1 & 1,8 \\
\hline Berlin & 346,8 & 0,8 & 3,4 & 0,3 & 1,0 & 0,4 & 1,7 & 0,9 & 3,5 & 0,6 & 2,3 & 0,5 & 1,8 \\
\hline Brandenburg & $1.168,8$ & 5,8 & 7,2 & 3,7 & 4,3 & 3,1 & 3,5 & 2,3 & 2,5 & 2,1 & 2,2 & 2,0 & 2,1 \\
\hline Mecklenburg-Vorpommern & 780,6 & 2,5 & 4,6 & 1,2 & 2,2 & $-1,1$ & $-1,9$ & 1,0 & 1,8 & 1,0 & 1,7 & 0,8 & 1,4 \\
\hline Sachsen & $1.131,4$ & 4,1 & 5,3 & 1,8 & 2,2 & 2,2 & 2,6 & 2,4 & 2,8 & 2,0 & 2,3 & 2,0 & 2,2 \\
\hline Sachsen-Anhalt & 923,9 & 3,7 & 5,8 & 3,7 & 5,6 & $-8,7$ & $-12,3$ & 1,6 & 2,4 & 1,3 & 1,8 & 1,1 & 1,5 \\
\hline Thüringen & 655,3 & 1,6 & 3,5 & 1,0 & 2,1 & 0,9 & 1,8 & 0,8 & 1,6 & 0,6 & 1,3 & 0,6 & 1,2 \\
\hline $\begin{array}{l}\text { Neue Bundesländer } \\
\text { (inkl. Berlin) }\end{array}$ & 5.007 & 18,4 & 5,4 & 11,6 & 3,2 & $-3,2$ & $-0,8$ & 9,0 & 2,4 & 7,7 & 2,0 & 6,9 & 1,8 \\
\hline Deutschland & 21.937 & 78,3 & 5,0 & 58,7 & 3,7 & 32,7 & 2,0 & 40,1 & 2,4 & 34,9 & 2,0 & 32,0 & 1,8 \\
\hline
\end{tabular}

${ }^{a}$ Quelle: Statistisches Bundesamt (2009)

${ }^{b}$ Quelle: PANTA RHEI REGIO Referenzszenario (9/2009)

\section{Zukünftige Siedlungsentwicklung}

Bisherige Projektionen der zukünftigen Flächeninanspruchnahme in Deutschland gehen aufgrund des demographischen Wandels von einer mehr oder weniger starken Reduktion gegenüber dem heutigen Niveau aus (vgl. Bundesamt für Bauwesen und Raumordnung 2007: 65; Bürkner/Berger/ Luchmann et al. 2007). All diesen Aussagen ist jedoch gemein, dass sie auf relativ einfachen Modellrechnungen fußen, die das komplexe Gefüge, welches für das Zustandekommen von Flächeninanspruchnahme verantwortlich ist, nur bedingt abbilden. Bisherige Bemühungen, die zukünftige Flächeninanspruchnahme im Rahmen des Modells Panta Rhei abzubilden, einem in einer Vielzahl von Anwendungen erprobten Instrument zur Analyse und Bewertung von umweltökonomischen Fragestellungen (Meyer/Bockermann/Ewerhart et al. 1998; Meyer/Bockermann/Ewerhart et al. 1999; Bach/Bork/Kohlhaas et al. 2001; Lutz 2004; Staiß/Kratzat/Nitsch et al. 2006, Lutz/Meyer/Nill et al. 2007; Lehr/Lutz 2008; Lutz/Meyer 2008), waren wiederum dadurch gekennzeichnet, dass der Grad der Regionalisierung unbefriedigend blieb (vgl. Ahlert/Klann/Lutz et al. 2005; Petschow/Zimmermann/Distelkamp et al. 2007). Für die Flächeninanspruchnahme gilt jedoch in besonderem Maße, dass sie auf regionaler Ebene - und hier insbesondere durch die regional stark disparat verlaufende Bevölkerungsentwicklung - determiniert wird (Jörissen/Coenen 2006; Siedentop/Heiland/Lehmann et al. 2007). Vor diesem
Hintergrund ist das Modell Panta Rhei im Bereich „Wohnen und Fläche“ um eine explizite Bottom-up-Modellierung auf Ebene der Landkreise und kreisfreien Städte erweitert worden (vgl. Ahlert/Klann/Lutz et al. 2005). Dieses neue Analyseinstrument für flächenpolitische Fragestellungen trägt die Bezeichnung Panta Rhei Regio (PRR). ${ }^{4}$

\subsection{Das Modell Panta Rhei Regio (PRR)}

Die Grundlagen der Flächenmodellierung in PRR liegen in der Identifizierung überwiegend nachfragebezogener Einflussfaktoren sowie der Einflüsse von Preisänderungen und deren konsequente Verknüpfung mit zu erwartenden gesamtwirtschaftlichen und regionalen Entwicklungen. ${ }^{5}$ Dabei sind die neu entwickelten regionalen Module eingegliedert in den Kontext weiterer gesamtwirtschaftlicher Modelle (INFORGE und LÄNDER ${ }^{6}$ ), die von der Gesell-

\footnotetext{
${ }^{4}$ Die Modellierung ist Ergebnis des Forschungsvorhabens „PANTA RHEI REGIO: Modellgestützter Dialog zur Siedlungs- und Verkehrsflächenentwicklung und Folgenabschätzung fiskalischer Maßnahmen auf nationaler und regionaler Ebene", das durch das Bundesministerium für Bildung und Forschung im Rahmen des Förderschwerpunkts „REFINA - Forschung für die Reduzierung der Flächeninanspruchnahme und ein nachhaltiges Flächenmanagement" finanziert wurde.

${ }^{5}$ Eine ausführliche Darstellung der Systemzusammenhänge in PRR findet sich in Distelkamp/Großmann/Hohmann et al. (2009).

${ }^{6}$ INFORGE (INterindustry FORecasting GErmany) ist ein nach Sektoren gegliedertes gesamtwirtschaftliches Modell für die Bundesrepublik Deutschland. Es wird seit über zehn Jahren regelmäßig
} 
schaft für Wirtschaftliche Strukturforschung (GWS) laufend aktualisiert werden. $\mathrm{Zu}$ den regionalen Modulen zählt im Wesentlichen die Modellierung der wirtschaftlichen Entwicklung aller 439 Kreise, das Wohnungsmodul, worin auf Grundlage der demographischen und wirtschaftlichen Entwicklung der regionale Wohnungsbau abgeleitet und für Deutschland aufgerechnet wird (Bottom-up-Ansatz) sowie ein Modul zur Ermittlung zukünftiger Wirtschaftsflächennachfrage in Deutschland insgesamt und in den Regionen. Alle Systemzusammenhänge sind empirisch fundiert, werden in einem konsistenten Datensatz zusammengeführt und für eine Projektion bis 2020 genutzt. Mit der Fokussierung auf Preiseinflüsse und nachfrageseitige Einflussfaktoren im Kontext der Flächeninanspruchnahme ist keineswegs die Aussage verbunden, dass weitere angebotsseitige Faktoren wie beispielsweise die positivplanerische Regulierung der Flächennutzung keinen Einfluss nehmen. Vielmehr wird im Referenzszenario unterstellt, dass sich diese Faktoren gegenüber dem historischen Zeitraum nicht verändern. Die im Folgenden vorgestellten Projektionen sind das Ergebnis eines im Herbst 2009 erstellten Referenzszenarios, in dem die Systemzusammenhänge auf Grundlage amtlicher Statistiken bis zum Berichtsjahr 2005 abgeleitet wurden.?

\subsection{Möglichkeiten und Grenzen des Modells}

Wie bereits erwähnt können angebotsseitige Faktoren, sofern sie sich nicht in Preisentwicklungen widerspiegeln, nur begrenzt berücksichtigt werden. Damit ist die Prognosegüte insbesondere auf regionaler Ebene eingeschränkt. Allgemein nimmt die Fundiertheit der Vorausberechnung mit zunehmendem Regionalisierungsgrad zu. Hier spielt auch die Verwertbarkeit des statistischen Ausgangmaterials eine größere Rolle. Im Bereich der Bevölkerungsentwicklung bestehen auf Kreisebene jene Beschränkungen, die auch für die Raumordnungsprognose gelten (Bundesamt für Bauwesen und Raumordnung 2009).

Darüber hinaus erfordert die Modellierung eine hohe Qualität und Verfügbarkeit des statistischen Ausgangsmate-

aktualisiert und wird bei verschiedenen Studien erfolgreich eingesetzt (vgl. Distelkamp/Hohmann/Lutz et al. 2003; Schnur/Zika 2009). Es stellt den ökonomischen Kern des Modells PANTA RHEI dar und ist damit auch zentraler Bestandteil von PRR. Das LÄNDER-Modell wurde zur Analyse und Prognose des Strukturwandels auf der Ebene der 16 Bundesländer entwickelt. Es ist direkt mit dem gesamtdeutschen Modell INFORGE gekoppelt und regionalisiert konsistent die dort auf der sektoralen Ebene ermittelten Ergebnisse für Wertschöpfung und Beschäftigung.

${ }^{7}$ Die hier angeführten Projektionsergebnisse unterscheiden sich teils von dem in Distelkamp/Großmann/Hohmann et al. (2009) dargestellten Ergebnisstand, da im Rahmen des REFINA-Projektes DoRiF (Designoptionen und Implementation von Raumordnungsinstrumenten zur Flächenverbrauchsreduktion) Verbesserungen der Modellierung der Wirtschaftsflächen mit entsprechenden Auswirkungen auf die Modellergebnisse erreicht werden konnten. rials. Die im Bereich der Flächenstatistik bekannten Ungenauigkeiten und Unplausibilitäten (vgl. Deggau 2006; Dosch 2008) schränken die Aussagekraft ein. Bei der Entwicklung des Modells standen primär die Abbildung der „tatsächlichen" Flächennutzung und ihr Wandel im Vordergrund. Die dafür notwendigen Bereinigungen führen mitunter dazu, dass Vergleiche mit der historischen Entwicklung bzw. mit neuen aktuellen Zahlen nur begrenzt aussagekräftig sind.

Weiterer Forschungs- bzw. Verbesserungsbedarf wird darüber hinaus auch im Bereich der Flächeninanspruchnahme der Wirtschaft gesehen. Hier konnten im Rahmen der bisherigen Arbeiten an PRR zwar regionale Disparitäten bei der Flächenintensität einzelner Wirtschaftsbereiche aufgedeckt werden. Die Hintergründe bzw. Einflussfaktoren, die für diesen Befund verantwortlich sind, blieben jedoch weitgehend ungeklärt, so dass für die Projektionen einfache Hypothesen zur Permanenz der regionalen Disparitäten in diesem Bereich formuliert werden mussten.

Trotz dieser zur kurzfristigen bzw. regionalen Prognosegüte eingestandenen Schwächen steht mit der ersten Modellversion ein System zur Verfügung, welches die Perspektive der Nachhaltigkeit der Siedlungsentwicklung in Deutschland aus unterschiedlichen Blickwinkeln beleuchten kann. Die auf Basis von PRR generierten Projektionen zur zukünftigen Flächeninanspruchnahme sind jedoch keinesfalls als Punktprognosen zu interpretieren, sondern zeigen empirisch begründete Entwicklungstendenzen auf, die einen fundierten Dialog über zukünftige Herausforderungen auf dem Weg zu einer nachhaltigen Flächennutzung ermöglichen. Im Rahmen eines Forschungsprojektes für das Bundesinstitut für Bau-, Stadt- und Raumforschung (BBSR) wird bis Sommer 2010 eine Aktualisierung und umfassende Weiterentwicklung von PRR erarbeitet, die sich explizit mit den in diesem Abschnitt angeführten Möglichkeiten und Grenzen des Modells kritisch auseinandersetzt und die (regionale) Prognosegüte verbessern wird.

\subsection{Perspektive 2020 - Entwicklung der Flächeninanspruchnahme}

Im Referenzszenario von PRR geht die Flächeninanspruchnahme für Siedlung und Verkehr von ehemals über 100 ha auf knapp unter 60 ha pro Tag zurück (vgl. Tab. 2, Projektion 2016 bis 2020). Auf Länderebene nehmen die Wachstumsdivergenzen nicht zu, obwohl weite Teile Ostdeutschlands von besonders starken Bevölkerungsrückgängen geprägt sind. In der Projektion steigt der Anteil der Gebäude- und Freiflächen an der gesamten Siedlungsflächenentwicklung im Vergleich zum letzten Vierjahreszeitraum (2004 bis 2008) deutlich an (vgl. Tab. 2 und 3). Dies ist größtenteils auf die Bereinigung der als unplausibel eingestuften Daten im Bereich der Erholungsflächen zurückzuführen. Darüber hinaus wird eine konstant hohe Flächeninanspruchnahme 
für den überörtlichen Verkehr erwartet, was sich besonders stark in den neuen Bundesländern äußert. Die Neuinanspruchnahme für Gebäude- und Freiflächen geht in Deutschland von in der jüngeren Vergangenheit durchschnittlich etwa 60 ha auf etwa 32 ha pro Tag zurück (vgl. Tab. 3). Der Schwerpunkt des Flächenverbrauchs liegt dabei in zunehmendem Maße auf dem Bereich Gebäude- und Freiflächen der Wirtschaft, da der Wohnungsbau voraussichtlich kontinuierlich an Bedeutung für die Flächeninanspruchnahme verlieren wird.

Die Wachstumsdivergenzen der Gebäude- und Freiflächen zwischen den Bundesländern gehen im Referenzszenario zwar gegenüber den historischen Beobachtungen zurück, bleiben jedoch über den gesamten Projektionshorizont bestehen. So wird etwa in Schleswig-Holstein, Bayern und Baden-Württemberg im Zeitraum von 2016 bis 2020 ein Wachstum der Gebäude- und Freiflächen von immer noch 2,5 \% erwartet. Dagegen fällt die Entwicklung in Niedersachsen, Nordrhein-Westfalen und RheinlandPfalz von ehemals über drei auf unter $2 \%$ zurück. In den neuen Bundesländern bleibt die Flächeninanspruchnahme in Sachsen und Brandenburg besonders hoch. Insgesamt sind im Jahr 2020 kaum noch wesentliche Wachstumsunterschiede zwischen den neuen und alten Bundesländern zu erkennen.

Auf der Ebene der Raumordnungsregionen zeigen sich im PRR-Referenzszenario deutlich mehr Unterschiede als auf Bundesländerebene (vgl. Abb. 1). Die räumlichen Muster der Flächeninanspruchnahme verändern sich nicht grundlegend gegenüber den historischen Beobachtungen. Stärkere Flächenexpansionen sind weiterhin im Bereich der Agglomerationsräume München und Stuttgart und deren Umfeld sowie in der Region Rhein-Neckar zu erwarten. Auch die flächenexpansiven Tendenzen in Nordwest-Niedersachsen und im Hamburger Großraum bleiben bestehen. Der Siedlungsdruck im Umland von Berlin, Leipzig und Dresden bleibt ebenfalls in ähnlicher Form erhalten. Besonders gering fällt hingegen die Flächeninanspruchnahme im Saarland und im Westen von Nordrhein-Westfalen aus. Vom Oberpfälzer Wald bis Weserbergland wird für weite Bereiche kaum Flächeninanspruchnahme erwartet. Dies gilt auch für viele ländliche Räume in Ostdeutschland. Diese Entwicklungsmuster sind weitgehend ein Spiegelbild der erwarteten wirtschaftlichen Dynamik und Bevölkerungsentwicklung
Abb. 1 Entwicklung der Gebäude- und Freifläche 2008 bis 2020 und Vergleich mit den historischen Entwicklungen. (Quelle: PRR-Referenzszenario 9/2009; Raumordnungsregionen 2008; Karte erstellt mit Regiograph)

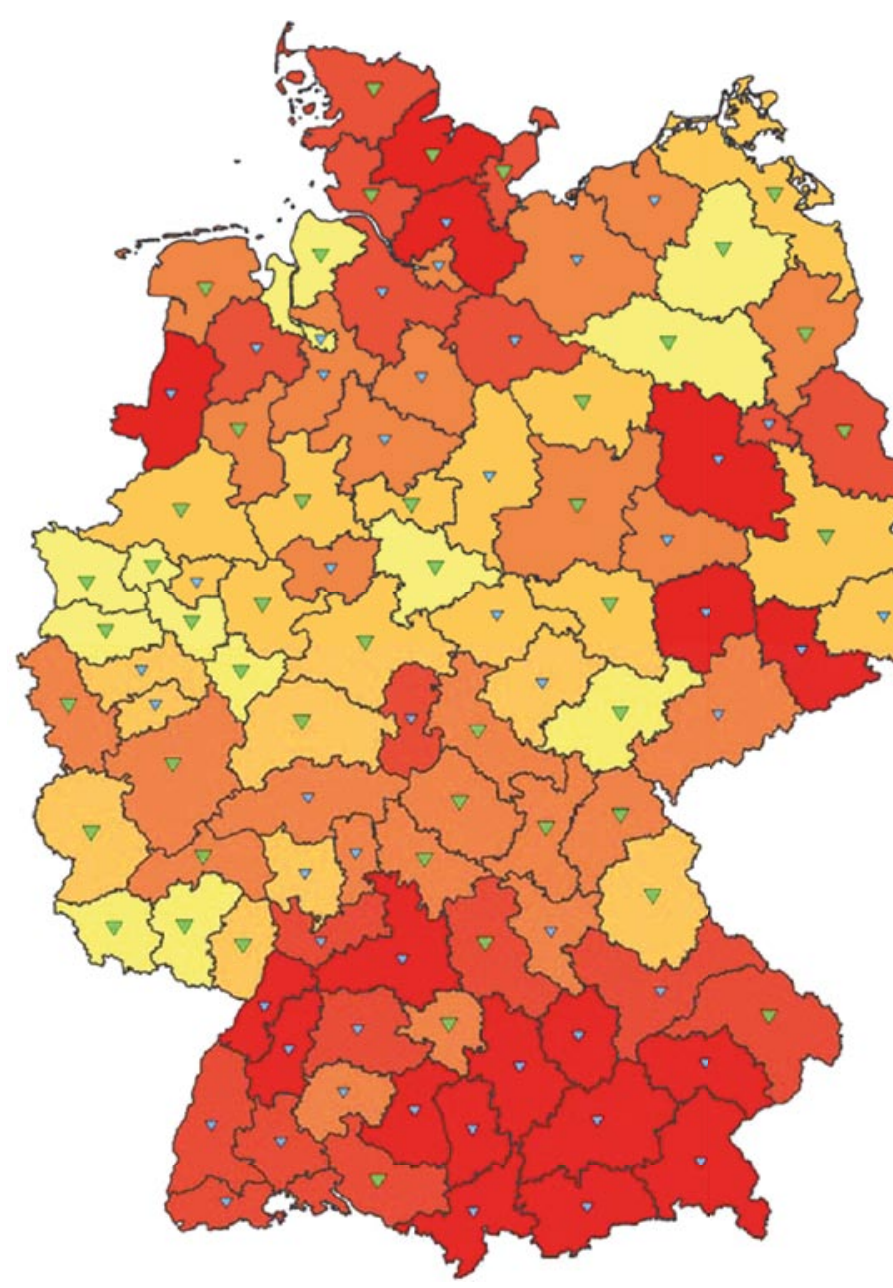
Wachstum 2008-2020
in \%

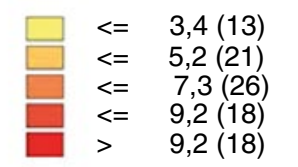

Veränderung der Flächeninanspruchnahme zw. 1996-2004 und 2008-2020

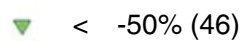

$\nabla \quad>=-50 \%(50)$ 
und sind zusätzlich ein Ergebnis des in der Vergangenheit beobachteten Umgangs mit freier Fläche in den Kreisen.

Die allgemeine Abschwächung des Siedlungswachstums findet nicht überall im gleichen Maße statt. Die blauen Dreiecke in Abb. 1 zeigen die Regionen, die zukünftig über $50 \%$ weniger Flächen in Anspruch nehmen werden. ${ }^{8}$ Dagegen geben die grünen Dreiecke eine geringe Veränderung der Flächeninanspruchnahme $(<-50 \%)$ an. Die Gegenüberstellung zeigt, dass eine Entschärfung des Siedlungsdrucks beispielsweise für weite Teile Süddeutschlands ausbleibt. Ähnlich ist es für die Regionen Dresden und Leipzig. Für Hamburg und sein weiteres Umland wird sich der Flächenverbrauch im bundesweiten Vergleich ebenfalls voraussichtlich nur leicht abschwächen. Für fast alle Regionen in Rheinland-Pfalz, Saarland und Nordrhein-Westfalen wird hingegen erwartet, dass der Rückgang der Flächeninanspruchnahme von Gebäude- und Freiflächen überdurchschnittlich ausfällt. Gleiches gilt für viele ländliche Räume nordwestlich von Berlin und in Vorpommern sowie weite Teile Sachsen-Anhalts und die Region Lausitz-Spreewald. Alle diese Regionen sind in besonderem Maße auch in Zukunft vom ökonomischen und demographischen Strukturwandel betroffen.

\section{Regionale Anpassungserfordernisse}

Die Bestimmung regionaler Anpassungserfordernisse bedingt eine Aufteilung des nationalen 30-ha-Ziels auf einzelne räumliche Einheiten. Konkrete und präzise Zielformulierungen für einzelne Gebietskörperschaften stellen allerdings eine große politische Herausforderung dar, da hierdurch Standards entstehen, die von den Normadressaten auch unmittelbar wahrgenommen werden. Generell sind quantitative Ziele wegen der Absolutheit der beinhalteten Beschränkung viel stärker Diskussionsgegenstand als qualitative Anforderungen zur Minimierung negativer ökologischer Folgen zukünftiger Siedlungsentwicklung. Dies dürfte einerseits an der mangelnden Transparenz qualitativer Beschränkungen und der damit sehr komplizierten und unsicheren Abschätzung ihrer Folgen liegen. Andererseits sind die wenig konkreten qualitativen Anforderungen zumeist sehr plausibel und damit leichter konsensfähig als mengenmäßige Beschränkungen. Dennoch werden Mengenziele für die Siedlungsentwicklung auch auf Bundesländer- und Regionalplanungsebene seit Jahren gefordert (Einig/Spiecker 2002; Bachmann 2005) oder in einigen Ländern wie beispielsweise in Baden-Württemberg mit dem

\footnotetext{
${ }^{8}$ Ein Vergleich auf dieser Ebene war für 1996 nur bis 2004 und nicht bis 2008 möglich, da zum Zeitpunkt der Auswertung die Daten auf Kreisebene für einige Bundesländer für 2008 noch nicht vorlagen.
}

3-ha-Ziel konkret formuliert (Nachhaltigkeitsbeirat BadenWürttemberg 2004).

Zur Verteilung des nationalen 30-ha-Ziels auf die einzelnen räumlichen Einheiten kann eine ganze Reihe von Verfahren mit sehr unterschiedlichen verteilungspolitischen Folgen herangezogen werden. Individuelle gesetzliche Selbstverpflichtungen der Länder, Regionen und Kommunen dürften sich zwar eher am erwarteten Flächenbedarf und an raumplanerischen Kriterien als an anderen Methoden orientieren, sind aber äußerst unrealistisch in der Umsetzung. Realistischer erscheinen Indikatoren, die für alle Teilräume gleichermaßen gelten und in einem gemeinsam erarbeiteten „Allokationsplan“ vereinbart werden. Die Anwendung eines einheitlichen Top-down-Verteilungsschlüssels dürfte insbesondere auf höheren föderalen Ebenen wie den Bundesländern oder Planungsregionen als sachgemäß empfunden werden und politisch durchsetzbar sein. Eine weitere Zielaufteilung auf einzelne Kommunen oder sogar Ortsteile mit einem für mehrere Länder bzw. Regionen geltenden Schlüssel wird dagegen mit großer Sicherheit auf harte politische Widerstände stoßen, wenn diese sich nicht klar an den Zielen und Grundsätzen der Raumordnung und der örtlichen Baulandnachfrage orientiert. Demzufolge sollte für ausreichend große Räume nach einem einheitlichen Schlüssel gesucht werden, der die allgemeine Zielvorgabe für Länder und Regionen plausibel übersetzt und dadurch auch auf den politischen Ebenen Akzeptanz finden kann.

Jedes Verfahren zur Zielaufteilung geht aber mit bestimmten Vor- und Nachteilen einher. Es ist daher nicht verwunderlich, dass bereits eine Vielzahl von Verteilungsschlüsseln diskutiert wurde (Krumm 2002; Jakubowski/Zarth 2003; Walz/Toussaint/Küpfer et al. 2005; Köck/Bizer/Einig et al. 2008; Henger/Schröter-Schlaack 2008). Vorgeschlagen wurden insbesondere flächenbezogene Indikatoren, die sich z. B. auf die Kataster-, Siedlungsfläche, Siedlungsentwicklung (vergangen und zukünftig) oder Bevölkerungs- und Siedlungsdichte beziehen. Geprüft wurden ebenso eine Reihe sozioökonomischer Kennzahlen wie Bevölkerung, Bevölkerungsentwicklung, Wirtschaftskraft und Erwerbstätige. Auch eine Verknüpfung bestimmter Top-down-Kriterien mit lokalen Flächenbedarfen und raumplanerischen Zielsetzungen fanden Berücksichtigung (z. B. Eigenentwicklungsindizes, Innenentwicklungspotenziale, Schutzgebietskategorien; vgl. z. B. Siedentop 2008).

Erfolgt eine Beurteilung nach den Kriterien Datenverfügbarkeit, Transparenz und Überprüfbarkeit, erscheint insbesondere eine Zielaufteilung anhand des Anteils der Bevölkerung in einem Teilraum an dem der gesamtdeutschen Bevölkerung attraktiv (Ostertag/Schleich/Ehrhart et al. 2010). Dieses Verfahren würde jedem Bundesbürger unabhängig von der aktuellen Siedlungsdichte den gleichen Umfang am Wachstum der Siedlungs- und Verkehrsflächen zugestehen. Alleine angewandt weist dieser Indikator jedoch 
erhebliche Plausibilitätsdefizite auf, da er den tatsächlichen Bedarf einer Gebietskörperschaft nicht abbilden kann und zu extremen Verteilungsmustern führt. Es besteht daher die Notwendigkeit, diesen Verteilungsschlüssel mit einem weiteren zu kombinieren. Zur Kombination mit der Bevölkerung drängt sich insbesondere die Katasterfläche eines Teilraums auf, da hierdurch Regionen aus Flächenländern mit geringer Bevölkerungsdichte wesentlich mehr Entwicklungspotenziale erhalten und die vermeintlich unerwünschten Verteilungsmuster eines Bevölkerungsschlüssels ausgeglichen werden. Im Vergleich zur nicht identisch erhobenen Siedlungsfläche liegen auch für kleine Teilräume exakte Flächendaten vor. Zusätzlich kann auf diese Weise stärker berücksichtigt werden, dass Räume mit geringer Dichte mehr Flächen für Infrastruktureinrichtungen benötigen. Neben diesen Vorzügen ist natürlich kritisch anzumerken, dass einer Zielaufteilung nach der Gemarkungsfläche jedweder Bezug zu bisherigen raumplanerischen Leitbildern fehlt und der Gesamtnachfrage nach Siedlungsflächen entgegen läuft.

Entscheidet man sich für die Anwendung dieser beiden Verteilungsschlüssel, dann bietet sich an, die beiden Indikatoren im Verhältnis 1:1 in einem gemischten Schlüssel miteinander zu verknüpfen. Mit diesem Bevölkerungs-Fläche-Indikator (BeFla-Indikator) werden Teilräume mit geringer Bevölkerungsdichte nicht zu stark benachteiligt und Teilräume mit hoher Dichte nicht zu stark bevorzugt (Henger/Schröter-Schlaack 2008). Für hinreichend große Teilräume liefert dieser Zuteilungsschlüssel nachvollziehbare und plausible Verteilungsmuster. Wie bereits diskutiert, könnte der BeFla-Indikator auf Ebene der Bundesländer und Planungsregionen als sinnvoller Orientierungswert Berücksichtigung finden. Ein bundeseinheitliches Verfahren muss zwar von örtlichen Gegebenheiten abstrahieren, bietet aber die Chance zur Formulierung und Umsetzung vergleichbarer normativer Nachhaltigkeitsindikatoren. Falls eine Verbindlichkeit der Zielaufteilung nicht gelingen sollte, bietet der Indikator die Möglichkeit zu einer Durchführung interregionaler Vergleiche und damit zu einer Bewusstseinsförderung der politischen Akteure auf Länder- und Regionsebene (vgl. u. a. Bizer/Sternberg 2001).

Im Folgenden wird daher der BeFla-Indikator vorgestellt und es werden die Anpassungserfordernisse für die einzelnen Teilräume diskutiert. Ausgangspunkt ist das 30ha-Reduktionsziel, welches eine Verringerung des Siedlungs- und Verkehrsflächenwachstums auf 30 ha pro Tag fordert. Hierfür werden die BeFla-Zielwerte mit den Werten der vergangenen und der prognostizierten Siedlungsentwicklung verglichen. Die Auswirkungen einer Zielaufteilung nach dem BeFla-Indikator werden auf den Ebenen der Bundesländer und Raumordnungsregionen betrachtet.

Der BeFla-Indikator berechnet sich durch die Mittelung des Flächen- und Bevölkerungsanteils eines Teilraums.

$$
x_{i}=\left(\frac{p_{i}}{P}+\frac{a_{i}}{A}\right) * \frac{X}{2}
$$

mit $\mathrm{x}_{\mathrm{i}}=$ Zuteilungsanteil eines Teilraums $\mathrm{i} ; \mathrm{X}=$ Flächensparziel; $p_{i}=$ Bevölkerungsanteil; $P=$ Gesamtbevölkerung; $\mathrm{a}_{\mathrm{i}}=$ Anteil an Katasterfläche; $\mathrm{A}=$ Gesamtfläche.

Beispielsweise hat Nordrhein-Westfalen einen Bevölkerungsanteil von $21,9 \%$ (17,9 von 82,0 Millionen Einwohnern, Stand 31.12.2008) und einen Flächenanteil von $9,5 \%$ an der Gesamtfläche Deutschlands $\left(34086 \mathrm{~km}^{2}\right.$ von $357104 \mathrm{~km}^{2}$ ). Der prozentuale Anteil am Flächensparziel entspricht dann nach dem BeFla-Indikator 15,7 \% für Nordrhein-Westfalen.

Wie in Kap. 2 beschrieben, bezieht sich der vorliegende Beitrag nicht alleine auf die gesamte Siedlungs- und Verkehrsflächenentwicklung, sondern ebenfalls auf die Entwicklung der Gebäude- und Freiflächen. Daher muss das Flächensparziel noch für Gebäude- und Freiflächen formuliert werden. Hierfür können die Anteile der Flächennutzungsarten am Zuwachs der Siedlungs- und Verkehrsfläche herangezogen werden. Wird der Zeitraum 1996 bis 2006 zugrunde gelegt, so entfällt auf die Gebäude- und Freiflächen ein Kontingent von 58,1 \% am Flächenziel. ${ }^{9}$ Das 30-ha-Ziel für Siedlungs- und Verkehrsflächen entspricht demzufolge 17,4 ha für die Gebäude- und Freiflächen. Ausgehend von diesem Reduktionsziel lassen sich die in Tab. 4 dokumentierten Teilziele für die einzelnen Bundesländer ableiten.

Die Zielwerte in Relation zu der durchschnittlichen Flächeninanspruchnahme in den Jahren 1996 bis 2008 ergeben die jeweiligen Anpassungserfordernisse beim Umgang mit Flächen aus heutiger Sicht. Im Bundesdurchschnitt muss sich demnach der Flächenverbrauch für Gebäude- und Freiflächen in den nächsten zehn Jahren um insgesamt knapp $70 \%$ gegenüber den historischen Werten reduzieren. Besonders deutlich fallen die Anpassungserfordernisse in den Bundesländern Schleswig-Holstein, Niedersachsen, Bayern und Brandenburg aus (vgl. Tab. 4). Die Ergebnisse aus der PRR-Projektion für den tatsächlichen in der Zukunft $\mathrm{zu}$ leistenden Anpassungsumfang liefern für einige Länder deutliche Unterschiede. Zunächst aber kann festgestellt werden, dass alle Länder - mit Ausnahme Bremens - über der BeFla-Zielvorgabe liegen. Die notwendigen Anpassungen sind aber insgesamt nicht mehr so groß, da das Gebäudeund Freiflächenwachstum von 56,6 (1996 bis 2008) auf 35,7 ha pro Tag (2008 bis 2020) um knapp $40 \%$ zurückgeht (vgl. Tab. 3). Die zukünftige Reduktionsverpflichtung für Gebäude- und Freiflächen entspricht daher nur noch $51,5 \%$. Dieser Rückgang ist vor allem in Nordrhein-Westfalen, Rheinland-Pfalz, Saarland und in Ostdeutschland (mit

\footnotetext{
${ }^{9}$ Der Zeitraum 1996-2006 wurde gewählt, da zum Zeitpunkt der Entstehung des vorliegenden Beitrags noch keine Daten für Gebäude- und Freiflächen vom 31.12.2008 vorlagen.
} 
Tab. 4 BeFla-Zielvorgaben und Anpassungserfordernisse für die Bundesländer

\begin{tabular}{|c|c|c|c|c|c|c|c|c|c|}
\hline \multirow[t]{2}{*}{ Bundesland } & \multicolumn{2}{|c|}{ Bevölkerung $^{\mathrm{a}}$} & \multicolumn{2}{|c|}{ Katasterfläche $^{\mathrm{a}}$} & \multicolumn{2}{|c|}{ BeFla-Zielvorgabe } & \multicolumn{3}{|c|}{ Red.-Verpflichtung GF in \% } \\
\hline & in Mio. & Anteil in $\%$ & in $\mathrm{km}^{2}$ & Anteil in $\%$ & $\begin{array}{l}\text { Anteil } \\
\text { in } \%\end{array}$ & $\begin{array}{l}\text { SuV ha } \\
\text { pro Tag }\end{array}$ & $\begin{array}{l}\text { GF ha pro } \\
\text { Tag }^{\mathrm{c}}\end{array}$ & $1996-2008$ & $2008-2020^{b}$ \\
\hline Schleswig-Holstein & 2,8 & 3,5 & 15.799 & 4,4 & 3,9 & 1,2 & 0,7 & $-83,8$ & $-70,4$ \\
\hline Hamburg & 1,8 & 2,2 & 755 & 0,2 & 1,2 & 0,4 & 0,2 & $-34,5$ & $-49,6$ \\
\hline Niedersachsen & 7,9 & 9,7 & 47.625 & 13,3 & 11,5 & 3,5 & 2,0 & $-78,7$ & $-62,4$ \\
\hline Bremen & 0,7 & 0,8 & 404 & 0,1 & 0,5 & 0,1 & 0,1 & $-37,7$ & 25,3 \\
\hline Nordrhein-Westfalen & 17,9 & 21,9 & 34.086 & 9,5 & 15,7 & 4,7 & 2,7 & $-61,1$ & $-18,7$ \\
\hline Hessen & 6,1 & 7,4 & 21.115 & 5,9 & 6,7 & 2,0 & 1,2 & $-53,4$ & $-37,7$ \\
\hline Rheinland-Pfalz & 4,0 & 4,9 & 19.853 & 5,6 & 5,2 & 1,6 & 0,9 & $-72,1$ & $-25,2$ \\
\hline Baden-Württemberg & 10,7 & 13,1 & 35.751 & 10,0 & 11,6 & 3,5 & 2,0 & $-71,5$ & $-62,6$ \\
\hline Bayern & 12,5 & 15,3 & 70.552 & 19,8 & 17,5 & 5,3 & 3,0 & $-77,4$ & $-60,8$ \\
\hline Saarland & 1,0 & 1,3 & 2.569 & 0,7 & 1,0 & 0,3 & 0,2 & $-63,1$ & $-25,8$ \\
\hline $\begin{array}{l}\text { Alte Bundesländer } \\
\text { (ohne Berlin) }\end{array}$ & 65,5 & 79,9 & 248.510 & 69,6 & 74,8 & 22,4 & 12,9 & $-72,8$ & $-53,5$ \\
\hline Berlin & 3,4 & 4,2 & 891 & 0,2 & 2,2 & 0,7 & 0,4 & $-21,9$ & $-41,8$ \\
\hline Brandenburg & 2,5 & 3,1 & 29.480 & 8,3 & 5,7 & 1,7 & 1,0 & $-76,7$ & $-54,5$ \\
\hline $\begin{array}{l}\text { Mecklenburg- } \\
\text { Vorpommern }\end{array}$ & 1,7 & 2,0 & 23.185 & 6,5 & 4,3 & 1,3 & 0,7 & $-15,9$ & $-22,4$ \\
\hline Sachsen & 4,2 & 5,1 & 18.418 & 5,2 & 5,1 & 1,5 & 0,9 & $-66,8$ & $-58,1$ \\
\hline Sachsen-Anhalt & 2,4 & 2,9 & 20.447 & 5,7 & 4,3 & 1,3 & 0,7 & & $-43,8$ \\
\hline Thüringen & 2,3 & 2,8 & 16.172 & 4,5 & 3,6 & 1,1 & 0,6 & $-44,0$ & $-5,4$ \\
\hline $\begin{array}{l}\text { Neue Bundesländer } \\
\text { (inkl. Berlin) }\end{array}$ & 16,5 & 20,1 & 108.594 & 30,4 & 25,2 & 7,6 & 4,4 & $-51,2$ & $-44,6$ \\
\hline Deutschland & 82,0 & & 357.104 & & 100,0 & 30,0 & 17,3 & $-69,4$ & $-51,5$ \\
\hline
\end{tabular}

auelle: Statistisches Bundesamt (Genesis Online Regional): Stichtag Bevölkerung: 31.12.2008, Stichtag Katasterfläche: 31.12 .2007

${ }^{b}$ Quelle: PANTA RHEI REGIO Referenzszenario (9/2009)

'Teilziel GF-Fläche

Einschränkung für Brandenburg und Sachsen) zu erwarten. Der Anpassungsdruck für diese Länder fällt folglich im Vergleich zu den amtlichen Statistiken deutlich geringer aus.

Abbildung 2 zeigt die Anpassungserfordernisse auf Ebene der Raumordnungsregionen in Prozent, wenn man die vom 30-ha-Ziel abgeleiteten „normativen“ BeFlaZielvorgaben in Relation zu den Projektionsergebnissen für Gebäude- und Freiflächen setzt. Die gelb markierten Regionen müssen keine Anpassungen in Zukunft vornehmen. Hierbei handelt es sich teilweise wiederum um die im vorherigen Abschnitt identifizierten Räume, die durch einen starken Bevölkerungsrückgang und eine schwache wirtschaftliche Entwicklung gekennzeichnet sind. Gleichzeitig sind diese Regionen entweder Agglomerationsräume (wie in Nordrhein-Westfalen) oder ländlich geprägte Räume (wie z. B. im Norden Brandenburgs) mit sehr hoher bzw. sehr niedriger Bevölkerungsdichte. Für diese Teilräume mit extremen Bevölkerungsdichten liefert der BeFla-Indikator relativ großzügige Zielvorgaben. Bei Kernstädten wird zwar der hohe Bevölkerungsanteil mit dem geringen Flächenanteil ausgeglichen. Dieser „Ausgleich“ führt aber nicht dazu, dass diese Regionen mit einem restriktiven Zielanteil bedacht werden. Umgekehrt gleicht der BeFlaIndikator zwar den hohen Flächenanteil mit dem geringen Bevölkerungsanteil in ländlich geprägten Regionen aus, aber in der Tendenz führt der Mischindikator trotzdem zu einer relativ großzügigen Zielvorgabe. Die stärksten
Anpassungen müssen die Regionen vornehmen, die auch in Zukunft eine weiterhin hohe Flächeninanspruchnahme zu erwarten haben (blau markiert). Wie zu Abb. 1 diskutiert, sind das vorrangig Räume mit einer hohen Wirtschafts- und Bevölkerungsentwicklung. Insgesamt zeigt die Gegenüberstellung der BeFla-Zielvorgaben mit der prognostizierten Siedlungsentwicklung, dass der Indikator plausible Zielvorgaben liefert, da Regionen mit starkem prognostizierten Siedlungswachstum zu Anpassungen gezwungen werden, während strukturschwache Regionen keine Einschränkungen ihrer Entwicklungsmöglichkeiten erfahren.

\section{Schlussfolgerungen}

Zusammengefasst lassen sich aus dem vorliegenden Beitrag drei wesentliche Schlussfolgerungen ziehen:

(1) Das zukünftig zu erwartende Siedlungsflächenwachstum macht den sparsamen Umgang mit natürlichen und naturnahen Flächen zu einer wesentlichen Zukunftsaufgabe räumlicher Planung.

(2) Diese Beobachtung unterstreicht die Notwendigkeit quantitativer Zielvorgaben und ihre Relevanz zur Umsetzung einer nachhaltigen Siedlungsentwicklung. Der in diesem Beitrag mit seinen regionalen Auswirkungen diskutierte BeFla-Indikator stellt eine Möglichkeit 
Abb. 2 Anpassungserfordernisse der Raumordnungsregionen bei der Flächeninanspruchnahme für Gebäude- und Freiflächen (GF-Flächen). (Quelle: PRR-Referenzszenario 9/2009; Raumordnungsregionen 2008; Karte erstellt mit Regiograph)

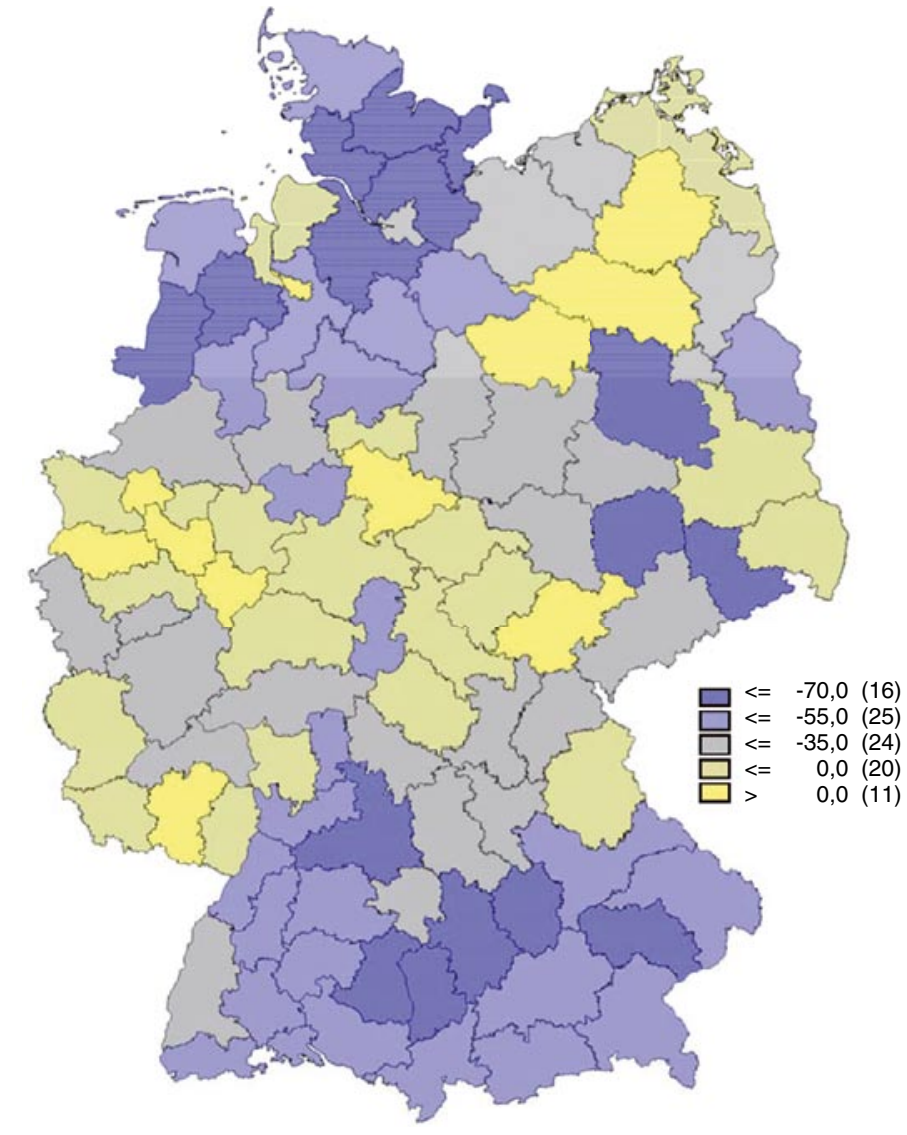

BeFla-Zielvorgabe in Relation zum Projektionsergebnis GFFlächen für die Jahre 2008 bis 2020 in \% dar, überörtliche Mengenziele auf regionale Teilgebiete aufzuteilen.

(3) Aus dieser Analyse lässt sich schließen, dass eine flexible Instrumentierung zur treffsicheren Begrenzung der zukünftigen Siedlungsflächenentwicklung erforderlich ist, um die großen regionalen Divergenzen des Wachstumsdrucks abzufedern und zu dämpfen.

Zu (1): Die Modellierung mit dem Prognosemodell PRR zeigt, dass zukünftig mit einem moderateren, aber stetigen Siedlungsflächenwachstum im Bundesdurchschnitt zu rechnen ist. Diese aus ökologischer, aber durchaus auch fiskalpolitischer Sicht positive Entwicklung wird insbesondere durch den demographischen Wandel und der damit geringer werdenden Nachfrage nach Wohnflächenentwicklung gestützt. Es bleibt allerdings festzuhalten, dass regional erhebliche Divergenzen in der Siedlungsentwicklung zu beobachten sein werden, die zu einem weiteren Anwachsen des Drucks auf die verbliebenen Freiflächen im Umland bestehender und weiterhin wachsender Agglomerationen führen wird. Hier wird das Problem einer flächenschonenden Siedlungsentwicklung nicht ohne veränderte Rahmenbedingungen gelöst werden können. Angesichts der bislang eher geringen Durchschlagskraft der Regionalplanung bei der Begrenzung des kommunalen Siedlungsflächenwachstums ist nicht damit $\mathrm{zu}$ rechnen, dass hier die bestehende
Instrumentierung für eine spürbare quantitative Reduzierung der Flächeninanspruchnahme ausreichen wird. Die Ergänzung des überwiegend qualitativ steuernden Instrumentariums und die Entwicklung und Stärkung quantitativer Steuerungsmöglichkeiten bleiben damit wesentliche Zukunftsaufgaben der überörtlichen Planung.

$\mathrm{Zu}$ (2): Ein Ansatzpunkt hierfür liegt in der Einführung quantitativer Zielvorgaben in die Regionalplanung. Mit der Verbindung etablierter qualitativer Lenkungsansätze mit Zielen zur höchstens möglichen Flächeninanspruchnahme kann wirksam einer beständigen Ausweitung der beanspruchten Flächen entgegengewirkt werden. Gleichzeitig zeigen sich aber aus den in diesem Beitrag unternommenen Überlegungen die großen Schwierigkeiten bei der Aufstellung und Verteilung quantitativer Wachstumsziele. Einerseits sind absolute Mengenziele der Siedlungsentwicklung per se schwer zu begründen, stellen sie doch das Flächensparen als das wichtigste Ziel raumordnerischer Steuerung in den Vordergrund. Das regionalplanerische Verständnis einer geordneten räumlichen Entwicklung richtet sich ebenso an anderen Zielgrößen aus, manche durchaus mit höherem Gewicht als das bloße Flächensparen. Erhebliche politische Widerstände sind zudem zu erwarten, wenn Mengenziele auf die Ebene der eigentlichen Regelungsadressaten, z. B. Regionen, Kreise und Gemeinden, aufgeteilt werden. Die Ausführungen in diesem Beitrag verdeutlichen den resul- 
tierenden Anpassungsdruck für die Regionen, wenn für eine regionale Aufteilung des 30-ha-Ziels auf die Regionen der BeFla-Indikator herangezogen wird. Andererseits wurde aus dem Vergleich zwischen geschätzter regionaler Siedlungsentwicklung nach PRR und Zuteilung an Siedlungsflächenwachstum nach BeFla-Indikator an die Regionen deutlich, dass mittels einfacher und transparenter Verteilungsschlüssel, die wiederum am ehesten vermittelbar sein dürften, eine befriedigende Annäherung an die zu erwartende Verteilung der räumlichen Siedlungsentwicklung nicht gelingt.

$\mathrm{Zu}$ (3): Daraus folgt, dass eine ergänzende Instrumentierung zur Steuerung der Siedlungsentwicklung nicht nur eine Stärkung der quantitativen Zielvorgaben in der räumlichen Planung umfassen sollte, sondern gleichzeitig eine weit reichende Flexibilität bei der Erreichung der Mengenvorgaben ermöglicht werden muss. Aus dem Kanon der in der Diskussion befindlichen Instrumente sticht unter diesem Gesichtspunkt der Ansatz handelbarer Flächenausweisungsrechte hervor, der genau dies zu erreichen verspricht (vgl. stellvertretend Köck/Bizer/Einig et al. 2008). Über die Handelbarkeit der Ausweisungsrechte wird ein Ventil geschaffen, das die stringenten Mengenvorgaben für einzelne Regelungsadressaten lockert. Kommunen oder Regionen, die eine für ihren Wachstumsdruck zu geringe Mengenzuteilung erhalten haben, können mit jenen Gemeinden und Regionen in einen Handel eintreten, die eine großzügige Ausstattung erhalten haben und ggf. zusätzliche Rechte nicht selbst in Anspruch nehmen wollen. Durch den so in Gang gesetzten Mechanismus erhält die Flächenausweisung einen Knappheitspreis und flächenschonende Siedlungsentwicklung wird über den Verkaufserlös der Zertifikate entlohnt. Dies dürfte einerseits $\mathrm{zu}$ einer verstärkten Beschäftigung mit den direkten und indirekten fiskalischen Folgen der Siedlungsentwicklung und ihrer Sinnhaftigkeit auf kommunaler Ebene führen und den Nachfragedruck nach weiterer Siedlungsflächenentwicklung zusätzlich zu den Auswirkungen des demographischen Wandels verringern. Gleichzeitig lockert die nachträgliche Handelbarkeit der Ausweisungsrechte die Verbindlichkeit der Mengenvorgabe und beugt damit einer erdrosselnden Wirkung einer stärker quantitativen Steuerung der Siedlungsentwicklung vor. Letztlich kann ein solcher Flächenhandel auch zu einer Partizipation weniger prosperierender Regionen am Siedlungs- und Wirtschaftswachstum wachsender Regionen führen und damit die gesellschaftliche Kohäsion erhöhen. Das Interesse der neuen Bundesregierung an einem entsprechenden Modellvorhaben handelbarer Rechte zur Steuerung der Flächennutzung unterstreicht die vorhandene Bereitschaft, das Instrument und seine Gestaltungsoptionen weiter zu untersuchen und einem Praxistest zu unterziehen (Bundesregierung 2009: 32).

\section{Literatur}

Ahlert, G.; Klann, U.; Lutz, C.; Meyer, B.; Wolter, M. I. (2005): Abschätzungen der Auswirkungen alternativer Bündel ökonomischer Anreizinstrumente zur Reduzierung der Flächeninanspruchnahme - Ziele, Maßnahmen, Wirkungen. Osnabrück. = GWS Discussion Paper 2005/5.

Apel, D.; Henckel, D.; Bunzel, A.; Floeting, H.; Henkel, M. J.; Kühn, G.; Lehmborck, M.; Sander, R. (1995): Flächen sparen, Verkehr reduzieren: Möglichkeiten zur Steuerung der Siedlungs- und Verkehrsentwicklung. Berlin. = Difu-Beiträge zur Stadtforschung, Bd. 16.

Bach, S.; Bork, C.; Kohlhaas, M.; Lutz, C.; Meyer, B.; Praetorius, B.; Welsch, H. (2001): Die ökologische Steuerreform in Deutschland: Eine modellgestützte Analyse ihrer Wirkungen auf Wirtschaft und Umwelt. Berlin/Heidelberg/New York.

Bachmann, G. (2005): Grenzen der Siedlungserweiterung? Was sich der Rat für Nachhaltigkeit vom „Ziel-30-ha“ verspricht. In: Informationen zur Raumentwicklung 4/5, 199-203.

Bizer, K.; Bunzel, A.; Cichorowski, G.; Rottmann, M. (2006): Instrumente und Akteure in der Flächenkreislaufwirtschaft. Expertise. Berlin/Darmstadt/Göttingen.

Bizer, K.; Cichorowski, G.; Henger, R.; Stephenson, N. (2009): Vom Leitbild der Flächenkreislaufwirtschaft zur Implementation. In: Zeitschrift für angewandte Umweltforschung, Sonderheft 16, 38-55.

Bizer, K.; Sternberg, R. (2001): Grundprobleme von Indikatorensystemen für Regionale Nachhaltigkeit. In: Raumforschung und Raumordnung 59 5/6, 381-391.

Bovet, J.; Köck, W. (2008): Kompetenzrechtliche Aspekte der Einführung eines Systems handelbarer Flächenausweisungsrechte. In: Köck, W.; Bizer, K.; Einig, K.; Hansjürgens, B.; Siedentop, S. (Hrsg.): Handelbare Flächenausweisungsrechte - Anforderungsprofil aus ökonomischer, planerischer und juristischer Sicht. Baden-Baden, 48-55.

Bürkner, H.-J.; Berger, O.; Luchmann, C.; Tenz, E. (2007): Der demographische Wandel und seine Konsequenzen für Wohnungsnachfrage, Städtebau und Flächennutzung. Erkner. = IRS Working Paper, Nr. 36.

Bundesamt für Bauwesen und Raumordnung (2007): Wohnungs- und Immobilienmärkte in Deutschland 2006. Bonn. = Berichte, $\mathrm{Bd}$. 27.

Bundesamt für Bauwesen und Raumordnung (2009): Raumordnungsprognose 2025/2050 - Bevölkerung, private Haushalte, Erwerbspersonen. Bonn. = Berichte, Bd. 29.

Bundesregierung (2002): Perspektiven für Deutschland: Unsere Strategie für eine nachhaltige Entwicklung. Bundesregierung, Presse- und Informationsamt. Berlin.

Bundesregierung (2009): Wachstum. Bildung. Zusammenhalt. Der Koalitionsvertrag zwischen CDU, CSU und FDP. 17. Legislaturperiode. Berlin.

Davy, B. (2009): Flächenhaushalt Reconsidered: Alternatives to the German Federal 30 Hectares Goal. In: van der Valk, A; van Dijk, T.. (Hrsg.): Regional Planning for Open Space. London, 279-300.

Deggau, M. (2006): Nutzung der Bodenfläche - Flächenerhebung 2004 nach Art der tatsächlichen Nutzung. In: Wirtschaft und Statistik 3, 212-219.

Distelkamp, M.; Großmann, A.; Hohmann, F.; Lutz, C.; Ulrich, P.; Wolter, M. I. (2009): Panta Rhei Regio - Ein Modellsystem zur Projektion der künftigen Flächeninanspruchnahme in Deutschland und zur Folgenabschätzung fiskalischer Maßnahmen. Osnabrück. $=$ GWS Discussion Paper 2009/7. 
Distelkamp, M.; Hohmann, F.; Lutz, C.; Meyer, B.; Wolter, M. I. (2003): Das IAB/INFORGE-Modell: Ein neuer ökonometrischer Ansatz gesamtwirtschaftlicher und länderspezifischer Szenarien. Nürnberg. = Beiträge zur Arbeitsmarkt- und Berufsforschung, Bd. 275.

Dosch, F. (2008): Siedlungsflächenentwicklung und Nutzungskonkurrenzen. In: Technikfolgenabschätzung - Theorie und Praxis 17, 2, 41-51.

Einig, K.; Spiecker, M. (2002): Die rechtliche Zulässigkeit regionalplanerischer Mengenziele zur Begrenzung des Siedlungs- und Verkehrsflächenwachstums. In: Zeitschrift für Umweltrecht, Sonderheft Flächenhaushaltspolitik 13, 150-157.

Henger, R.; Schröter-Schlaack, C. (2008): Designoptionen für den Handel mit Flächenausweisungsrechten in Deutschland. Göttingen. $=$ Land Use Economics and Planning - Discussion Paper, No. 08-02.

Jakubowski, P.; Zarth, M. (2003): Nur noch 30 Hektar Flächenverbrauch pro Tag: Vor welchen Anforderungen stehen die Regionen? In: Raumforschung und Raumordnung 60, 3, 185-197.

Jörissen, J.; Coenen, C. (2006): TA-Projekt Reduzierung der Flächeninanspruchnahme. Ziele, Maßnahmen, Wirkungen. Berlin. $=\mathrm{TAB}$ Arbeitsbericht Nr. 98.

Köck, W.; Bizer, K.; Einig, K.; Hansjürgens, B.; Siedentop, S. (Hrsg.) (2008): Handelbare Flächenausweisungsrechte - Anforderungsprofil aus ökonomischer, planerischer und juristischer Sicht. Baden-Baden.

Krumm, R. (2002): Die Baulandausweisungsumlage als ökonomisches Steuerungsinstrument einer nachhaltigkeitsorientierten Flächenpolitik. Tübingen. $=$ IAW Discussion Paper, Nr. 7.

Lehr, U.; Lutz, C. (2008): Employment effects within the Climate Change Policy Framework. In: European Parliament. (Hrsg.): Competitive distortions and leakage in a world of different carbon prices. Strasbourg, 44-60.

Lutz, C. (2004): Panta Rhei. In: Forum für Energiemodelle und Energiewirtschaftliche Systemanalysen in Deutschland. (Hrsg.): Energiemodelle zum europäischen Klimaschutz. Der Beitrag der deutschen Energiewirtschaft. Münster, 693-744. = Umwelt- und Ressourcenökonomik, Bd. 22.

Lutz, C.; Meyer, B. (2008): Beschäftigungseffekte des Klimaschutzes in Deutschland. Untersuchungen zu gesamtwirtschaftlichen Auswirkungen ausgewählter Maßnahmen des Energie- und Klimapakets. Dessau-Roßlau. $=$ Umweltbundesamt, Climate Change $13 / 08$.

Lutz, C.; Meyer, B.; Nill, J.; Schleich, J. (2007): Windows of Opportunity for Radical Technological Change in Steel Production and the Influence of $\mathrm{CO} 2$ Taxes. In: Lehmann-Waffenschmidt, M. (Hrsg.): Innovations Towards Substainability: Conditions and Consequences. Heidelberg, 3-17.

Meyer, B.; Bockermann, A.; Ewerhart, G.; Lutz, C. (1998): Modellierung der Nachhaltigkeitslücke. Eine umweltökonometrische Analyse. Heidelberg.
Meyer, B.; Bockermann, A.; Ewerhart, G.; Lutz, C. (1999): Marktkonforme Umweltpolitik: Wirkungen auf Luftschadstoffemissionen, Wachstum und Struktur der Wirtschaft. Berlin.

Nachhaltigkeitsbeirat Baden-Württemberg (2004): Neue Wege zu einem Flächenmanagement in Baden-Württemberg. Stuttgart.

Ostertag, K.; Schleich, J.; Ehrhart, K.-M.; Goebes, L.; Müller, J.; Seifert, S.; Küpfer, C. (2010): Neue Instrumente für weniger Flächenverbrauch. Der Handel mit Flächenausweisungszertifikaten im Experiment. Karlsruhe.

Petschow, U.; Zimmermann, T.; Distelkamp, M.; Lutz, C. (2007): Wirkungen fiskalischer Steuerungsinstrumente auf Siedlungsstrukturen und Personenverkehr vor dem Hintergrund der Nachhaltigkeitsziele der Bundesregierung. Berlin/Osnabrück.

Ring, I. (2008): Compensating Municipalities for Protected Areas: Fiscal Transfers for Biodiversity Conservation in Saxony, Germany. In: Gaia - Ecological Perspectives for Science and Society 17, S1, 143-151.

Schnur, P.; Zika, G. (Hrsg.) (2009): Das IAB/INFORGE-Modell: Ein sektorales makro-ökonometrisches Projektions- und Simulationsmodell zur Vorausschätzung des längerfristigen Arbeitskräftebedarfs. Nürnberg.

Siedentop, S. (2008): Anforderungen aus raumordnungsplanerischer Sicht. In: Köck, W.; Bizer, K.; Einig, K.; Hansjürgens, B.; Siedentop, S. (Hrsg.): Handelbare Flächenausweisungsrechte - Anforderungsprofil aus ökonomischer, planerischer und juristischer Sicht. Baden-Baden, 110-158.

Siedentop, S.; Heiland, S.; Lehmann, I.; Schauerte-Lüke, N. (2007): Nachhaltigkeitsbarometer Fläche. Bonn. = Forschungen des BMVBS und BBR, Nr. 130.

Staiß, F.; Kratzat, M.; Nitsch, J.; Lehr, U.; Edler, D.; Lutz, C. (2006): Wirkungen des Ausbaus erneuerbarer Energien auf den deutschen Arbeitsmarkt. Gutachten im Auftrag des Bundesministeriums für Umwelt, Naturschutz und Reaktorsicherheit (BMU). Berlin.

Statistisches Bundesamt (2009): Land- und Forstwirtschaft, Fischerei - Bodenfläche nach Art der tatsächlichen Nutzung 2008. Wiesbaden.$=$ Fachserie 3, Reihe 5.1.

Umweltbundesamt (2003): Reduzierung der Flächeninanspruchnahme durch Siedlung und Verkehr. Berlin. $=$ Umweltbundesamt-Text 90/03.

Umweltbundesamt (2004): Flächenverbrauch, ein Problem mit wirtschaftlichen Folgen. Umweltbundesamt-Hintergrundpapier. Berlin.

Walz, R.; Toussaint, D.; Küpfer, C.; Sanden, J. (2005): Gestaltung eines Modells handelbarer Flächenausweisungskontingente unter Berücksichtigung ökologischer, ökonomischer, rechtlicher und sozialer Aspekte - Abschlussbericht. Umweltforschungsplan des Bundesministeriums für Umwelt, Naturschutz und Reaktorsicherheit. Berlin.

Wissenschaftlicher Beirat Bodenschutz (2002): Ohne Boden bodenlos. Berlin. 